\title{
Unil
}

UNIL | Université de Lausanne

Unicentre

$\mathrm{CH}-1015$ Lausanne

http://serval.unil.ch

Year : 2013

\section{Two Cases of Interferon-Alpha-Induced Sarcoidosis Koebnerized along Venous Drainage Lines: New Pathogenic Insights and Review of the Literature of Interferon-Induced Sarcoidosis}

\author{
Buss Guillaume
}

Buss Guillaume, 2013, Two Cases of Interferon-Alpha-Induced Sarcoidosis Koebnerized along Venous Drainage Lines: New Pathogenic Insights and Review of the Literature of Interferon-Induced Sarcoidosis

Originally published at: Thesis, University of Lausanne

Posted at the University of Lausanne Open Archive http://serval.unil.ch

Document URN : urn:nbn:ch:serval-BIB_E989ECBF89DF8

\section{Droits d'auteur}

L'Université de Lausanne attire expressément l'attention des utilisateurs sur le fait que tous les documents publiés dans I'Archive SERVAL sont protégés par le droit d'auteur, conformément à la loi fédérale sur le droit d'auteur et les droits voisins (LDA). A ce titre, il est indispensable d'obtenir le consentement préalable de l'auteur et/ou de l'éditeur avant toute utilisation d'une oeuvre ou d'une partie d'une oeuvre ne relevant pas d'une utilisation à des fins personnelles au sens de la LDA (art. 19, al. 1 lettre a). A défaut, tout contrevenant s'expose aux sanctions prévues par cette loi. Nous déclinons toute responsabilité en la matière.

\section{Copyright}

The University of Lausanne expressly draws the attention of users to the fact that all documents published in the SERVAL Archive are protected by copyright in accordance with federal law on copyright and similar rights (LDA). Accordingly it is indispensable to obtain prior consent from the author and/or publisher before any use of a work or part of a work for purposes other than personal use within the meaning of LDA (art. 19, para. 1 letter a). Failure to do so will expose offenders to the sanctions laid down by this law. We accept no liability in this respect. 


\section{UNIVERSITE DE LAUSANNE - FACULTE DE BIOLOGIE ET DE MEDECINE}

Département de Médecine

Service d'immunologie et allergie

\section{Two Cases of Interferon-Alpha-Induced Sarcoidosis Koebnerized along Venous Drainage Lines: New Pathogenic Insights and Review of the Literature of Interferon-Induced Sarcoidosis}

\section{THESE}

préparée sous la direction du Professeur M. Gilliet, Médecin-chef

et présentée à la Faculté de biologie et de médecine de l'Université de Lausanne pour l'obtention du grade de

DOCTEUR EN MEDECINE

par

Guillaume BUSS

Médecin diplômé de la Confédération Suisse

Originaire de Kirchberg (BE)

Lausanne 


\section{Unil}

UNIL | Université de Lausanne

Faculté de biologie et de médecine

Ecole Doctorale

Doctorat en médecine

\section{Imprimatur}

Vu le rapport présenté par le jury d'examen, composé de

Directeur de thèse Monsieurle Professeur Michel Gilliet

Co-Directeur de thèse

Expert

Directrice de l'Ecole Madame le Professeur Stephanie Clarke doctorale

la Commission MD de l'Ecole doctorale autorise l'impression de la thèse de

\section{Monsieur Guillaume Buss}

intitulée

Two Cases of Interferon-Alpha-Induced Sarcoidosis Koebnerized along Venous Drainage Lines: New Pathogenic Insights and Review of the Literature of Interferon-Induced Sarcoidosis

Lausanne, le 18 février 2014

pour Le Doyen

de la Faculté de Biologie et de Médecine

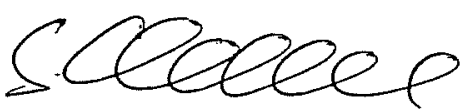

Madame le Professeur Stephanie Clarke Directrice de l'Ecole doctorale 


\title{
Two Cases of Interferon-Alpha-Induced \\ Sarcoidosis Koebnerized along Venous Drainage Lines: New Pathogenic Insights and Review of the Literature of Interferon-Induced Sarcoidosis
}

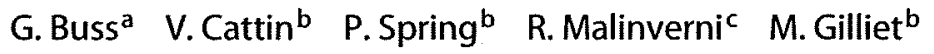 \\ aDivision of Immunology and Allergology and 'bepartment of Dermatology, University Hospital Center and \\ University of Lausanne, Lausanne, and 'Internal Medicine Department, Hôpital Neuchâtelois, Neuchâtel, \\ Switzerland
}

\section{Key Words}

Hepatitis $C \cdot$ Interferon-a $\cdot$ Intravenous drug user - Koebner phenomenon - Sarcoidosis . Venous drainage lines

\begin{abstract}
Sarcoidosis is a systemic granulomatous disorder of unknown origin commonly affecting the lung, the lymphoid system and the skin. We report here two cases of cutaneous sarcoidosis in two former intravenous drug users following interferon (IFN)- $a$ and ribavirin therapy for chronic hepatitis C. Both patients developed skin sarcoidosis along venous drainage lines of both forearms, coinciding with the areas of prior drug injections. The unique distribution of the skin lesions suggests that tissue damage induced by repeated percutaneous drug injections represents a trigger for the local skin manifestation of sarcoidosis. Interestingly, skin damage was recently found to induce the local expression IFN- $a$, a well-known trigger of sarcoidosis in predisposed individuals. Here we review the literature on sarcoidosis elicited in the context of IFN- $a$ therapy and propose a new link between the endogenous expression of IFN-a and the induction of disease manifestations in injured skin.
\end{abstract}

○ 2013 S. Karger AG, Basel

\section{Introduction}

Sarcoidosis is a multiple granulomatous disorder of unknown origin affecting the lung ( $>90 \%$ of cases), the lymphoid system $(33 \%)$, the liver $(50-80 \%)$, the eyes $(11-83 \%)$ and the skin (25\%) [1]. Interferon (IFN) $-\alpha$ is commonly used for treatment of chronic hepatitis $C$ because of its antiviral and immunomodulatory activities in combination with ribavirin [2]. We report one case of sarcoidosis limited to the skin and one case of systemic sarcoidosis with cutaneous involvement, both of them koebnerized along venous drainage lines in two former intravenous drug users treated with IFN- $\alpha$ and ribavirin for chronic hepatitis C.

\section{Case Reports}

\section{Case 1}

A 36-year-old man with a history of intravenous drug use and chronic hepatitis $C$ was started on a 48-week course of pegylated IFN and ribavirin. The patient had injected heroin into the veins of both forearms on daily basis for several years. However, he said he had stopped any drug consumption for more than 6 months be- fore starting the treatment. Chest X-ray before treatment was normal and abdominal sonography showed mild hepatomegaly. At week 30 of IFN- $\alpha$ and ribavirin therapy, painless, firm, erythematous skin papules developed in a linear distribution along the cephalic and the median antebrachial veins of both forearms, former areas of drug injections (fig. 1). The patient did not complain of itching, and no other site was involved. He had no history of trauma, infection or vaccination, and there was no family history of similar skin diseases. A skin biopsy revealed dermal inflammatory infiltrates with the presence of numerous noncaseating granulomas (fig. 2). No foreign material was detected by polarization light microscopy. This histologic finding along with the clinical picture was consistent with skin sarcoidosis. Furthermore, we found elevated serum levels of angiotensinconverting enzyme and mediastinal, retroperitoneal and axillary adenopathies, consistent with a systemic involvement of the sarcoidosis. Topical corticosteroids were administered and antiviral therapy was continued to maximize the chance of cure

G. Buss and V. Cattin contributed equally to this work.

\section{KARGER \\ E-Mail kargerekargercom}

\section{S. Karger AG, Basel} 1018-8665/13/2264-0289\$38.00/0

www.karger.com/drm

\section{Michel Gilliet, MD}

Department of Dermatology

University Hospital Center and University of Lausanne

CH-1011 Lausanne (Switzerland)

E-Mail michel.gilliet @ chuv.ch 
Fig. 1. Clinical aspect of skin sarcoidosis along forearm veins of patient $1(\mathbf{a}, \mathbf{b})$ and patient 2 (c, d). a, c At presentation. b, d After recovery.

Fig. 2. HE staining of tissue sections showing numerous sarcoid granulomas in the dermis of patients 1 (a) and 2 (b). Immunostaining with anti-CD123 antibody (c) revealed numerous pDCs in the inflammatory dermal infiltrate between the granulomas of patient 2 (some of which are indicated by black arrows).

from hepatitis C. At week 37, the patient developed polyuria and polydipsia in the setting of severe hypercalcemia and mild cholestasis, which has been described in systemic sarcoidosis [3]. Because IFN- $a$ and ribavirin therapy has been associated with the development of systemic sarcoid-
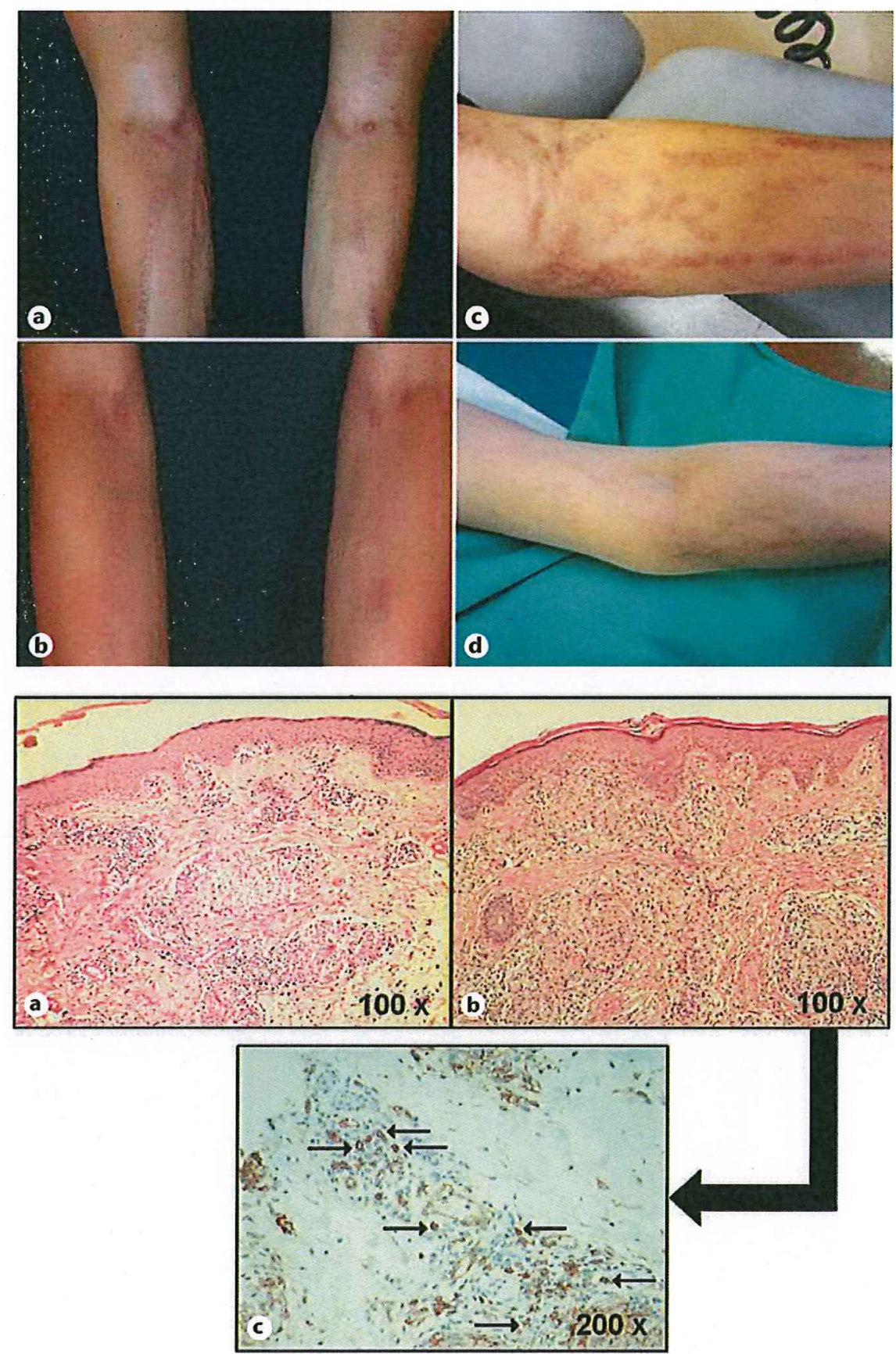

osis $[4,5]$, this treatment was discontinued and we introduced a short course of systemic steroids (prednisone $\mathbf{3 0} \mathrm{mg} /$ day) as recommended in symptomatic hypercalcemia [6]. This led to a rapid regression of skin lesions (fig. 1) and resolution of hypercalcemia and cholestasis. Prednisone was progressively reduced and stopped after 2 months. No relapse of the cutaneous or systemic sarcoidosis was observed. Despite the incomplete IFN and ribavirin treatment of 37 weeks instead of 48 weeks, the patient remained without evidence of hepatitis Cvirus (HCV) viremia after 6 months. 
Case 2

A 42-year-old woman with a history of intravenous drug use and genotype 6 chronic hepatitis $C$ was started on a 48 week course of pegylated IFN and ribavirin. The patient had injected drugs intravenously into both forearms on a daily basis for several years. She told us that she had stopped any drug consumption 5 years before starting the treatment. After 16 weeks of IFN- $\alpha$ and ribavirin therapy, the patient developed painless, nonpruriginous, firm, erythematous skin papules in a linear distribution along the veins of the forearms and of the dorsal aspect of the hands bilaterally, former areas of drug injections (fig. 1). Complete examination revealed no other cutaneous lesions. She had no history of trauma, infection or vaccination and there was no family history of similar skin diseases. A skin biopsy revealed dermal noncaseating granulomatous inflammatory infiltrates, containing multinucleated giant cells (fig. 2). No foreign material was detected by polarization light microscopy. This histologic finding along with the clinical picture was consistent with skin sarcoidosis. Serum levels of angiotensin-converting enzyme, blood chemistry and chest X-ray were normal and excluded a systemic involvement of the sarcoidosis. In the absence of a systemic sarcoidosis, we did not introduce systemic treatment and IFN and ribavirin therapy was continued for a total of 48 weeks. Skin lesions spontaneously disappeared rapidly after completion of IFN- $a$ and ribavirin therapy. The patient remained without evidence of $H C V$ viremia 6 months after the end of the IFN- $\alpha$ and ribavirin therapy.

\section{Discussion}

Sarcoidosis is a multisystem disorder of unknown cause. It commonly affects young and middle-aged adults and frequently presents with bilateral hilar lymphadenopathy, pulmonary infiltration, and ocular and skin lesions. The liver, spleen, lymph nodes, salivary glands, heart, nervous system, muscles, bones, and other organs may also be involved [7]. Although the exact cause of sarcoidosis remains unknown, it is thought to represent an exaggerated immune response to a yet unknown antigenic stimulus leading to the formation of granulomas. These granulomas are characterized by a predominance of CD $4+T$ helper cells

IFN-a-Induced Sarcoidosis Koebnerized along Venous Drainage Lines
(TH), producing large amounts of TH1type cytokines IFN- $\gamma$ and IL-2. Macro phages and dendritic cells are also activat ed to produce proinflammatory cytokines such as IL-1, TNF- $a$, and IL-12. By contrast, relatively few 'TH2 are found in the granulomas, confirming that this is a THImediated disease $[8,9]$.

Here we report two cases of sarcoidosis triggered by IFN- $\alpha$ and ribavirin therapy in patients with chronic hepatitis C. IFN- $\alpha$ is a potent immune stimulator that is used for the treatment of infectious and neoplastic diseases. IFN- $a$ was shown to induce the activation and maturation of dendritic cells into potent antigen-presenting cells, and to directly stimulate $\mathrm{T}$ cell activation and $\mathrm{TH} 1$ polarization. Furthermore, IFN- $\alpha$ differentiates immature $B$ cells into antibody-secreting plasma cells and promotes cytotoxicity of NK cells [10]. Ribavirin is a nucleoside analogue and was found to potentiate IFN- $\alpha$ expression potentially via Toll-like receptor (TLR) 7 and TLR8 $[11,12]$, providing an explanation for why the combination therapy of IFN- $\alpha$ plus ribavirin may be more efficacious in treating hepatitis $\mathrm{C}$ [13]. It is not surprising that this Th1-promoting therapy exacerbates sarcoidosis in predisposed individuals.

The first association between IFN-a and sarcoidosis was reported in 1993 [14]. Since then, 136 cases of IFN-induced sarcoidosis were reported (table 1). A systematic review of this literature revealed that there was no major sex or age bias: $59 \%$ of patients were female and the patients' age ranged from 23 to 76 years (with a median of 50.4 years). Our review of 138 cases (including our 2 patients) revealed that cutaneous manifestations of sarcoidosis were evident in $49 \%$ of the patients treated with IFN- $a$, whereas only half as many $(25 \%)[1]$ of the sarcoidosis patients had skin in volvement in the absence of IFN- $\alpha$ treatment. This is consistent with a previous report of IFN-induced sarcoidosis observing that in $50 \%$ of cases, skin involvement is the first manifestation of sarcoidosis, and that in $20 \%$ of these cases sarcoidosis remains confined to the skin [15]. The onset of IFN$\alpha$-induced sarcoidosis varies from 15 days to 30 months after the beginning of treatment [16]. Ninety-five percent of the cases with IFN- $\alpha$-induced sarcoidosis have been reported to improve or remit after discontinuation or a decrease in the dosage of the drug (table 1).

Interestingly, $83 \%$ of patients receiving IFN- $\alpha$ therapy for the treatment of hepatitis
C had IFN-induced sarcoidosis, while only $16 \%$ of patients with proliferative disorders receiving adjuvant IFN- $\alpha$ therapy had the disease. Several reasons may account for these differences: (1) hepatitis $C$ patients may have received higher doses of IFN- $a$, although, due to a lack of protocol details in most reports, no formal conclusion can be drawn; (2) in most antiviral protocols, IFN- $a$ is given in combination with ribavirin, which may represent an additional trigger of endogenous IFN- $\alpha$ via TLR7 and TLR8 as described above, and (3) the HCV itself may trigger the expression of endog. enous IFN- $\alpha$ and sarcoidosis as suggested by some reports describing sarcoidosis in untreated hepatitis $C$ patients $[17,18]$.

The Koebner phenomenon (KP) represents a typical characteristic of cutaneous sarcoidosis. In our review, we found that in $29 \%$ of cases skin sarcoidosis was triggered by a classical KP (table 1). Typical triggers of the KP in sarcoidosis are foreign material present in tattoos [19-21], synthetic fillers used for cosmetic procedures [22], or talc injected in the context of intravenous drug abuse $[23,24]$. Scars are also a typical trigger of KP and sarcoidosis $[25,26]$, although most of the time foreign particles are undetectable in cutaneous biopsies, namely in 51 (78\%) of 65 patients with scar sarcoidosis [25]. Similarly in our two cases, we did not find any foreign body material in the tissue samples by polarization light microscopy. It is possible that the repeated venipuncture over several years has resulted in tissue injury, chronic subclinical inflammation and scar formation, which koebnerized the cutaneous sarcoidosis despite the absence of any venous puncture for several months or even years. Interestingly, endogenous IFN- $\alpha$ was recently found to be expressed in wounded skin upon mechanical or chemical injury and chronic skin inflammation in psoriasis [27]. The endogenous IFN- $a$ was found to be produced by a subset of dendritic cells called plasmacytoid dendritic cells (pDCs), which are usually absent from the skin but rapidly infiltrate wounded skin. At this site, pDCs recognize host-derived nucleic acids released by dying cells into the injured tissue via TLR7 and TLR9, exclusively expressed by the pDCs [28]. This process ultimately leads to the production of a large quantity of IFN-a, which initiates wound healing. It is possible that in our two patients, the repeated trauma to the skin may have triggered a sustained skin infiltration by PDCs and their activation to produce 
Table 1. IFN-induced sarcoidosis reported $[5,13,14$, for a review $15,16,17,20,32-88]$

\begin{tabular}{|c|c|c|c|c|c|c|}
\hline Authors & Age/sex & $\begin{array}{l}\text { Indication } \\
\text { for IFN }\end{array}$ & Organ involvement & Treatment & Situation & Outcome \\
\hline Lee et al. [24] & $39 / \mathrm{M}$ & $\mathrm{HCV}$ & $\begin{array}{l}\text { lymphadenopathy } \\
\text { linear forearm papules }\end{array}$ & IFN-a + R & $\begin{array}{l}\text { discontinuation } \\
\text { of topical steroid }\end{array}$ & $\begin{array}{l}\text { resolution } \\
\text { ( } 2 \text { weeks) }\end{array}$ \\
\hline Bonnet et al. [89] & $-1-$ & $\mathrm{HCV}$ & lung & IFN- $\boldsymbol{\alpha}$ & suspension & improvement \\
\hline Godoy et al. [90] & $-1-$ & HCV & lung, skin & IFN-a + R & & resolution \\
\hline Godoy et al. [90] & $45 / \mathrm{M}$ & $\mathrm{HCV}$ & lung, skin & IFN $-a+R$ & & resolution \\
\hline Alazemi and Campos [91] & $42 / \mathrm{M}$ & $\mathrm{HCV}$ & $\begin{array}{l}\text { lymphadenopathy } \\
\text { lung } \\
\text { subcutaneous nodules }\end{array}$ & IFN-a+R & discontinuation & $\begin{array}{l}\text { resolution } \\
\text { (12 months) }\end{array}$ \\
\hline Iwashita et al. [92] & $72 / \mathrm{F}$ & $\mathrm{HCV}$ & $\begin{array}{l}\text { skin } \\
\text { lymphadenopathy } \\
\text { uveitis, heart }\end{array}$ & IFN-a+R & $\begin{array}{l}\text { discontinuation } \\
\text { of oral steroid }\end{array}$ & improvement \\
\hline Atluri et al. [19] & $45 / \mathrm{M}$ & $\mathrm{HCV}$ & tattoo papules & IFN- $\alpha+R$ & oral steroid & resolution \\
\hline Bitetto et al. [93] & $64 / \mathrm{F}$ & $\mathrm{HCV}$ & nasolabial folds & IFN- $a+R$ & oral steroid & $\begin{array}{l}\text { resolution } \\
\text { (few weeks) }\end{array}$ \\
\hline Meller et al. [94] & $55 / \mathrm{F}$ & $\mathrm{HCV}$ & nodules/papules & IFN-a + R & $\begin{array}{l}\text { discontinuation } \\
\text { of topical steroid }\end{array}$ & resolution \\
\hline Honsová et al. [95] & $-1-$ & $\mathrm{HCV}$ & skin & IFN-a+R & discontinuation & resolution \\
\hline Sanchez-Ruano et al. [96] & $43 / \mathrm{M}$ & $\mathrm{HCV}$ & lung, skin & IFN-a+R & & \\
\hline Perera and Calonje [21] & $54 / \mathrm{M}$ & $\mathrm{HCV}$ & $\begin{array}{l}\text { lung } \\
\text { lymphadenopathy } \\
\text { ulcerated tattoo site nodules }\end{array}$ & IFN-a+R & topical steroid & improvement \\
\hline Perez-Gala et al. [26] & $59 / \mathrm{F}$ & $\mathrm{HCV}$ & skin & IFN-a + R & topical steroid & $\begin{array}{l}\text { resolution } \\
\text { ( } 5 \text { months) }\end{array}$ \\
\hline Song and Das [97] & $-/ \mathrm{F}$ & $\mathrm{HCV}$ & nodules & IFN-a+R & & \\
\hline Adla et al. [98] & $44 / \mathrm{M}$ & $\mathrm{HCV}$ & liver & IFN-a + R & & \\
\hline Doycheva et al. [99] & $49 / \mathrm{F}$ & $\mathrm{HCV}$ & $\begin{array}{l}\text { ocular lesions } \\
\text { renal failure }\end{array}$ & IFN-a + R & $\begin{array}{l}\text { decreased } \\
\text { oral steroid } \\
\text { topical steroid }\end{array}$ & $\begin{array}{l}\text { resolution } \\
\text { ( } 6 \text { months) }\end{array}$ \\
\hline Doycheva et al. [99] & $37 / \mathrm{M}$ & $\mathrm{HCV}$ & ocular lesions & IFN- $a+R$ & $\begin{array}{l}\text { decreased } \\
\text { topical steroid }\end{array}$ & resolution \\
\hline Doycheva et al. [99] & $65 / \mathrm{F}$ & $\mathrm{HCV}$ & ocular lesions & IFN-a+R & $\begin{array}{l}\text { decreased } \\
\text { topical steroid }\end{array}$ & resolution \\
\hline Yan et al. $[100]$ & $23 / \mathrm{F}$ & $\mathrm{HCV}$ & $\begin{array}{l}\text { ocular lesions } \\
\text { lymphadenopathy }\end{array}$ & IFN-a + R & topical steroid & resolution \\
\hline Lopez et al. [101] & $55 / \mathrm{M}$ & $\mathrm{HCV}$ & $\begin{array}{l}\text { lung } \\
\text { lymphadenopathy } \\
\text { nodules }\end{array}$ & IFN- $\alpha+R$ & none & $\begin{array}{l}\text { resolution } \\
\text { (6 months) }\end{array}$ \\
\hline Petousi and Thomas [102] & $30 / \mathrm{F}$ & $\begin{array}{l}\text { multiple } \\
\text { sclerosis }\end{array}$ & $\begin{array}{l}\text { lung } \\
\text { lymphadenopathy }\end{array}$ & IFN $-\alpha+\beta$ & $\begin{array}{l}\text { discontinuation } \\
\text { of oral steroid }\end{array}$ & $\begin{array}{l}\text { resolution } \\
\text { (1 month) }\end{array}$ \\
\hline Chakravarty et al. [103] & $39 / \mathrm{F}$ & $\begin{array}{l}\text { multiple } \\
\text { sclerosis }\end{array}$ & $\begin{array}{l}\text { lung } \\
\text { papules/nodules }\end{array}$ & IFN- $\beta$ & $\begin{array}{l}\text { discontinuation } \\
\text { of oral steroid } \\
\text { hydroxychloro- } \\
\text { quine } \\
\text { methotrexate }\end{array}$ & $\begin{array}{l}\text { resolution } \\
\text { (6 months) }\end{array}$ \\
\hline Carbonelli et al. [104] & $35 / \mathrm{M}$ & $\begin{array}{l}\text { multiple } \\
\text { sclerosis }\end{array}$ & $\begin{array}{l}\text { lung, liver } \\
\text { lymphadenopathy }\end{array}$ & IFN- $\beta$ & & \\
\hline O'Reilly et al. [105] & $52 / \mathrm{F}$ & $\begin{array}{l}\text { multiple } \\
\text { sclerosis }\end{array}$ & $\begin{array}{l}\text { lung } \\
\text { lymphadenopathy }\end{array}$ & IFN- $\beta$ & discontinuation & $\begin{array}{l}\text { resolution } \\
\text { ( } 6 \text { months) }\end{array}$ \\
\hline Novoa et al. [106] & $54 / \mathrm{F}$ & $\mathrm{HCV}$ & $\begin{array}{l}\text { lung, liver } \\
\text { papules/nodules }\end{array}$ & IFN-a+R & discontinuation & resolution \\
\hline Albaker [107] & $48 / \mathrm{M}$ & $\mathrm{HCV}$ & $\begin{array}{l}\text { hypercalcemia } \\
\text { lung } \\
\text { lymphadenopathy }\end{array}$ & IFN $-\alpha+R$ & discontinuation & resolution \\
\hline
\end{tabular}


Table 1 (continued)

\begin{tabular}{|c|c|c|c|c|c|c|}
\hline Authors & Age/sex & $\begin{array}{l}\text { Indication } \\
\text { for IFN }\end{array}$ & Organ involvement & Treatment & Situation & Outcome \\
\hline Oudghiri et al. [30] & $47 / \mathrm{F}$ & $\mathrm{HCV}$ & $\begin{array}{l}\text { lung, CNS } \\
\text { papules }\end{array}$ & IFN- $\alpha+R$ & $\begin{array}{l}\text { discontinuation } \\
\text { of oral steroid }\end{array}$ & death \\
\hline North and Mully [108] & $52 / \mathrm{M}$ & melanoma & $\begin{array}{l}\text { lymphadenopathy } \\
\text { papules/nodules }\end{array}$ & IFN- $\boldsymbol{\alpha}$ & discontinuation & $\begin{array}{l}\text { resolution } \\
(6 \text { months })\end{array}$ \\
\hline Morley et al. [109] & $67 / \mathrm{F}$ & $\mathrm{HCV}$ & ocular lesions & IFN- $\alpha+R$ & oral steroid & $\begin{array}{l}\text { resolution } \\
\text { (12 months) }\end{array}$ \\
\hline Sionidou et al. [110] & $36 / \mathrm{M}$ & $\begin{array}{l}\text { polycythemia } \\
\text { vera }\end{array}$ & $\begin{array}{l}\text { lung } \\
\text { lymphadenopathy }\end{array}$ & IFN- $\alpha$ & $\begin{array}{l}\text { discontinuation } \\
\text { of oral steroid }\end{array}$ & \\
\hline Cardoso et al. [111] & $43 / \mathrm{M}$ & $\mathrm{HCV}$ & $\begin{array}{l}\text { lung } \\
\text { lymphadenopathy } \\
\text { papules/nodules }\end{array}$ & IFN- $\alpha+R$ & discontinuation & improvement \\
\hline Benzagmout et al. [31] & $47 / F$ & $\mathrm{HCV}$ & $\begin{array}{l}\text { CNS, lung } \\
\text { subcutaneous nodules }\end{array}$ & IFN- $\mathbf{a}+\mathrm{R}$ & $\begin{array}{l}\text { discontinuation } \\
\text { of systemic steroid }\end{array}$ & death \\
\hline Gayet et al. [112] & $57 / \mathrm{F}$ & $\mathrm{HCV}$ & $\begin{array}{l}\text { lung } \\
\text { lymphadenopathy } \\
\text { subcutaneous nodules }\end{array}$ & IFN- $\boldsymbol{\alpha}+\mathrm{R}$ & none & $\begin{array}{l}\text { resolution } \\
\text { (6 months) }\end{array}$ \\
\hline Heinzerling et al. [113] & $50 / \mathrm{M}$ & melanoma & $\begin{array}{l}\text { lymphadenopathy } \\
\text { lung, scar sarcoid }\end{array}$ & IFN- $\alpha$ & discontinuation & resolution \\
\hline Heinzerling et al. [113] & $47 / \mathrm{M}$ & melanoma & $\begin{array}{l}\text { lymphadenopathy } \\
\text { lung }\end{array}$ & IFN- $\alpha$ & discontinuation & resolution \\
\hline Heinzerling et al. [113] & $47 / \mathrm{M}$ & melanoma & $\begin{array}{l}\text { lymphadenopathy } \\
\text { lung }\end{array}$ & IFN- $\alpha$ & discontinuation & $\begin{array}{l}\text { resolution } \\
\text { (2 months) }\end{array}$ \\
\hline Pelletier et al. [18] & $60 / \mathrm{M}$ & melanoma & $\begin{array}{l}\text { lung } \\
\text { subcutaneous nodules }\end{array}$ & IFN- $\alpha$ & discontinuation & resolution \\
\hline Rodriguez-Lojo et al. [114] & $60 / \mathrm{F}$ & $\mathrm{HCV}$ & subcutaneous nodules & IFN- $\mathfrak{a}+\mathbf{R}$ & discontinuation & $\begin{array}{l}\text { resolution } \\
\text { ( } 2 \text { months) }\end{array}$ \\
\hline Faurie et al. [115] & $59 / \mathrm{M}$ & $\mathrm{HCV}$ & $\begin{array}{l}\text { lymphadenopathy } \\
\text { lung }\end{array}$ & IFN- $a+R$ & none & resolution \\
\hline Faurie et al. [115] & $65 / F$ & $\mathrm{HCV}$ & $\begin{array}{l}\text { lymphadenopathy } \\
\text { lung }\end{array}$ & IFN- $\alpha+R$ & none & resolution \\
\hline Faurie et al. [115] & $49 / \mathrm{F}$ & $\mathrm{HCV}$ & $\begin{array}{l}\text { lymphadenopathy } \\
\text { lung } \\
\text { subcutaneous nodules }\end{array}$ & IFN- $\alpha$ & discontinuation & resolution \\
\hline Faurie et al. [115] & $76 / \mathrm{F}$ & $\mathrm{HCV}$ & $\begin{array}{l}\text { uveitis } \\
\text { subcutaneous nodules }\end{array}$ & IFN-a + R & oral steroid & improvement \\
\hline Faurie et al. [115] & $56 / F$ & $\mathrm{HCV}$ & $\begin{array}{l}\text { lymphadenopathy } \\
\text { lung }\end{array}$ & IFN-a + R & oral steroid & resolution \\
\hline Le Bras et al. [116] & $54 / \mathrm{F}$ & $\mathrm{HCV}$ & $\begin{array}{l}\text { lymphadenopathy } \\
\text { lung } \\
\text { subcutaneous nodules }\end{array}$ & IFN- $\alpha+\mathbf{R}$ & oral steroid & $\begin{array}{l}\text { resolution } \\
\text { ( } 7 \text { months) }\end{array}$ \\
\hline Shuja et al. [23] & $59 / \mathrm{M}$ & $\mathrm{HCV}$ & linear forearm papules & IFN- $\alpha+\mathbf{R}$ & none & \\
\hline Zampino et al. [117] & $59 / F$ & $\mathrm{HCV}$ & nodule & IFN- $\alpha+\mathrm{R}$ & surgery & resolution \\
\hline Descamps et al. [22] & $48 / \mathrm{F}$ & $\mathrm{HCV}$ & $\begin{array}{l}\text { lung } \\
\text { papules/nodules } \\
\text { filler site reaction }\end{array}$ & IFN- $\boldsymbol{\alpha}+\mathrm{R}$ & oral steroid & resolution \\
\hline Descamps et al. [22] & $64 / \mathrm{F}$ & $\mathrm{HCV}$ & $\begin{array}{l}\text { lymphadenopathy } \\
\text { lung } \\
\text { subcutaneous nodules }\end{array}$ & IFN- $\alpha+R$ & $\begin{array}{l}\text { discontinuation } \\
\text { of oral steroid }\end{array}$ & $\begin{array}{l}\text { resolution } \\
\text { ( } 3 \text { months) }\end{array}$ \\
\hline Current report & $36 / \mathrm{M}$ & $\mathrm{HCV}$ & $\begin{array}{l}\text { lymphadenopathy } \\
\text { hypercalcemia } \\
\text { linear forearm papules }\end{array}$ & IFN- $\alpha+R$ & $\begin{array}{l}\text { discontinuation } \\
\text { of oral steroid }\end{array}$ & $\begin{array}{l}\text { resolution } \\
(2 \text { months })\end{array}$ \\
\hline Current report & $42 / \mathrm{F}$ & $\mathrm{HCV}$ & linear forearm papules & IFN $-a+R$ & topical steroid & $\begin{array}{l}\text { resolution } \\
\text { (6 months) }\end{array}$ \\
\hline
\end{tabular}


IFN- $\alpha$, triggering the local manifestation of sarcoidosis. The skin IFN- $\alpha$ levels may be further enhanced by the systemic IFN- $\alpha$ levels and, particularly, by ribavirin, which may further trigger activation of skin $\mathrm{pDCs}$ via TLR7. Our hypothesis is supported by the finding of numerous pDCs in the dermal infiltrate surrounding the sarcoid granulomas of patient 2 (fig. 2 ).

Finally, regarding management of IFNinduced sarcoidosis in HCV infection, we provide a decision algorithm (fig. 3): IFN discontinuation should be based on the extent of sarcoidosis. For instance, antiviral therapy could be continued in skin-limited or asymptomatic stage I pulmonary sarcoidosis, especially if the antiviral therapy has been introduced for more than 6 months. Maintaining treatment to maximize hepatitis $\mathrm{C}$ remission has to be evaluated jointly with hepatologists. Systemic steroids should be considered with caution in symptomatic IFN-induced sarcoidosis, because it may increase the viral load [29]. Moreover, most cases resolve spontaneously after drug discontinuation. Nevertheless, we recommend aggressive treatment of neurologic, ocular, cardiac and renal sarcoidosis or hypercalcemia, because death $[30,31]$, severe loss of vision, fatal arrhythmias, or insidious renal damage may ensue without therapy.

\section{Conclusion}

We report two cases of cutaneous sarcoidosis manifested by linear erythematous skin papules along forearm veins in two former intravenous drug users treated by IFN- $\alpha$ and ribavirin for chronic hepatitis $C$. In the literature we found two other cases with a similar distribution $[23,24]$. Because skin injury was recently found to induce local IFN- $\alpha$ expression by infiltrating $\mathrm{pDCs}$ and IFN- $\alpha$ is a well-known trigger of sarcoidosis in predisposed individu-

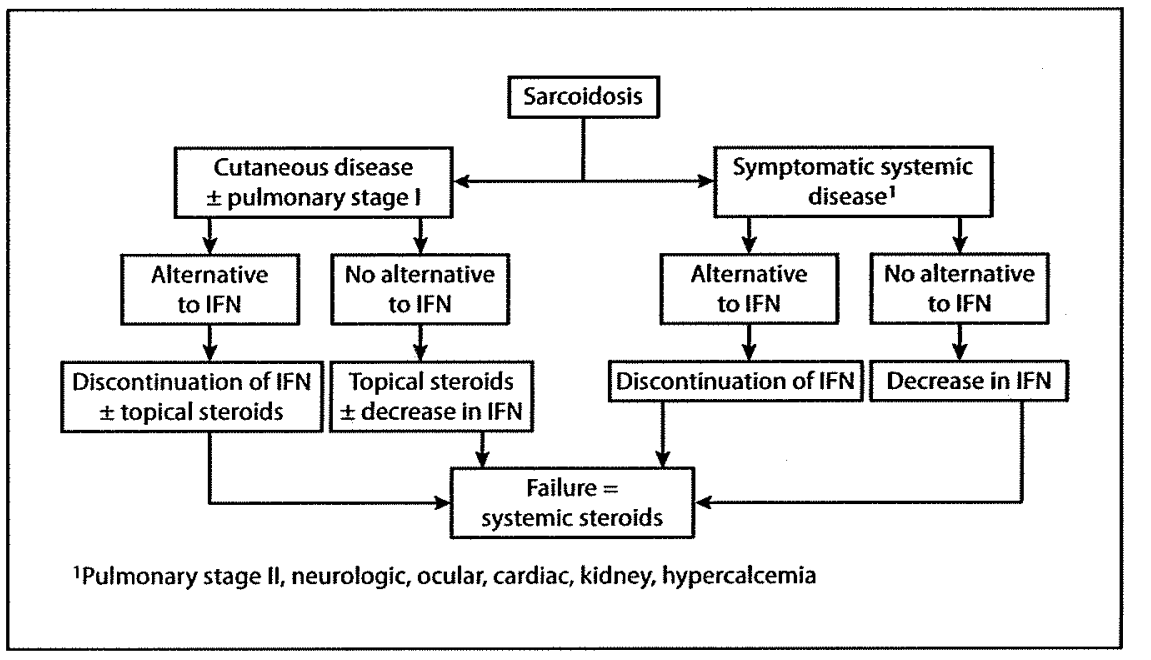

Fig. 3. Decision algorithm for IFN-induced sarcoidosis: IFN discontinuation should be based on the extent of sarcoidosis. For instance, antiviral therapy could be continued in skin-limited or asymptomatic stage I pulmonary sarcoidosis, especially if the antiviral therapy has been introduced for more than 6 months. Maintaining treatment to maximize hepatitis $\mathrm{C}$ remission has to be evaluated jointly with hepatologists. Systemic steroids should be considered with caution in symptomatic interferon-induced sarcoidosis, because it may increase the viral load [29]. Moreover, most cases resolve spontaneously after drug discontinuation. Nevertheless, we recommend aggressive treatment of neurologic, ocular, cardiac and renal sarcoidosis or hypercalcemia, because death $[30,31]$, severe loss of vision, fatal arrhythmias, or insidious renal damage may ensue without therapy.

als, we propose that our patients' specific skin manifestations along venous drainage lines may result from the additional IFN- $\alpha$ expression in previously injured skin. Moreover, it was shown that ribavirin therapy may further enhance IFN- $\alpha$ expression in previously wounded skin. Identification of pDCs surrounding granulomatous inflammation in our tissue samples seems to be an argument in that direction, supported by the absence of foreign body material detected by polarization light microscopy.
This condition may represent a key point in the understanding of the physiopathological mechanisms leading to scar sarcoidosis. Further investigations need to be performed to confirm this hypothesis.

\section{Disclosure Statement}

The authors have no conflicts of interest to declare.

\section{References}

1 Costabel $U$, Hunninghake GW: ATS/ERS/ WASOG statement on sarcoidosis. Sarcoidosis Statement Committee. American Thoracic Society. European Respiratory Society. World Association for Sarcoidosis and Other Granulomatous Disorders. Eur Respir J 1999; 14:735-737.

2 Bellobuono A, Mondazzi L, Tempini S, Silini E, Vicari F, Ideo G: Ribavirin and interferonalpha combination therapy vs interferon-alpha alone in the retreatment of chronic hepa- titis C: a randomized clinical trial. J Viral Hepat 1997;4:185-191.

3 Holmes J, Lazarus A: Sarcoidosis: extrathoracic manifestations. Dis Mon 2009;55:675-692.

4 Martins EV, Gaburri AK, Gaburri D, Sementilli A: Cutaneous sarcoidosis: an uncommon side effect of pegylated interferon and ribavirin use for chronic hepatitis C. Case Rep Gastroenterol 2009;3:366-371.

5 Ubina-Aznar E, Fernandez-Moreno N, Rivera-Irigoin R, Navarro-Jarabo JM, Garcia-
Fernandez G, Perez-Aisa A, Vera-Rivero F, Fernandez-Perez F, Moreno-Mejias P, Mendez-Sanchez I, de Sola-Earle C, Sanchez-Cantos A: Pulmonary sarcoidosis associated with pegylated interferon in the treatment of chronic hepatitis C (in Spanish). Gastroenterol Hepatol 2005;28:450-452.

6 Londner C, Zendah I, Freynet O, Carton Z, Dion G, Nunes H, Valeyre D: Treatment of sarcoidosis (in French). Rev Med Interne 2011;32:109-113. 
7 Statement on sarcoidosis. Joint Statement of the American Thoracic Society (ATS), the European Respiratory Society (ERS) and the World Association of Sarcoidosis and Other Granulomatous Disorders (WASOG) adopted by the ATS Board of Directors and by the ERS Executive Committee, February 1999. Am J Respir Crit Care Med 1999;160:736755.

8 Robinson BW, Mclemore TL, Grystal RG: Gamma interferon is spontaneously released by alveolar macrophages and lung $\mathrm{T}$ lymphocytes in patients with pulmonary sarcoidosis. J Clin Invest 1985;75:1488-1495.

9 Baumer 1, Zissel G, Schlaak M, Muller Quernheim J: Th1/Th2 cell distribution in pulmonary sarcoidosis. Am J Respir Cell Mol Biol 1997;16:171-177.

10 Parronchi P, Mohapatra S, Sampognaro S, Giannarini L, Wahn U, Chong P, Mohapatra S, Maggi E, Renz H, Romagnani S: Effects of interferon-alpha on cytokine profile, $\mathrm{T}$ cell receptor repertoire and peptide reactivity of human allergen-specific T cells. Eur J Immunol 1996;26:697-703.

11 Tam RC, Pai B, Bard J, Lim C, Averett DR Phan UT, Milovanovic T: Ribavirin polarizes human ' $\mathrm{T}$ cell responses towards a type $1 \mathrm{cy}$ tokine profile. J Hepatol 1999;30:376-382.

12 Vollmer J, Rankin R, Hartmann $H$, Jurk $M$, Samulowitz U, Wader T, Janosch A, Schetter C, Krieg AM: Immunopharmacology of CpG oligodeoxynucleotides and ribavirin. Antimicrob Agents Chemother 2004;48:2314-2317.

13 Hurst EA, Mauro T: Sarcoidosis associated with pegylated interferon alfa and ribavirin treatment for chronic hepatitis $\mathrm{C}$ : a case report and review of the literature. Arch Dermatol 2005;141:865-868.

14 Blum L, Serfaty L, Wattiaux MJ, Picard O, Cabane J, Imbert JC: Nodules hypodermiques sarcoïdosiques au cours d'une hépatite virale C traitée par interféron alpha $2 b$. Rev Med Interne 1993;14:1161.

15 Fantini F, Padalino C, Gualdi G, Monari P, Giannetti A: Cutaneous lesions as initial signs of interferon alpha-induced sarcoidosis: report of three new cases and review of the literature. Dermatol Ther 2009;22(suppl 1):S1S7.

16 Cogrel O, Doutre MS, Marliere V, BeylotBarry M, Couzigou P, Beylot C: Cutaneous sarcoidosis during interferon alfa and ribavirin treatment of hepatitis $\mathrm{C}$ virus infection: two cases. Br J Dermatol 2002;146:320-324.

17 Ramos-Casals M, Mana J, Nardi N, Brito-Zeron $P$, Xaubet $A$, Sanchez-Tapias JM, Cervera R, Font J, Group HS: Sarcoidosis in patients with chronic hepatitis $C$ virus infection: analysis of 68 cases. Medicine (Baltimore) 2005. 84:69-80.

18 Pelletier F, Manzoni P, Jacoulet P, Humbert $P$, Aubin F: Pulmonary and cutaneous sarcoidosis associated with interferon therapy for melanoma. Cutis 2007;80;441-445.
19 Atluri D, Iduru S, Veluru C, Mullen K: A levitating tattoo in a hepatitis $C$ patient on treatment. Liver Int 2010;30:583-584.

20 Toulemonde A, Quereux G, Dreno B: Sarcoidosis granuloma on a tattoo induced by interferon alpha (in French). Ann Dermatol Venereol 2004;131:49-51.

21 Perera GK, Calonje E: Systemic sarcoidosis presenting in a tattooed man undergoing treatment for hepatitis C. Clin Exp Dermatol 2006;31:387-389.

22 Descamps V, Landry J, Frances C, Marinho E, Ratziu V, Chosidow O: Facial cosmetic filler injections as possible target for systemic sarcoidosis in patients treated with interferon for chronic hepatitis C: two cases. Dermatology 2008;217:81-84.

23 Shuja F, Kavoussi SC, Mir MR, Jogi RP, Rosen $T$ : Interferon induced sarcoidosis with cutaneous involvement along lines of venous drainage in a former intravenous drug user. Dermatol Online J 2009;15:4.

24 Lee YB, Lee II, Park HJ, Cho BK, Oh ST: Interferon-alpha induced sarcoidosis with cutaneous involvement along the lines of venous drainage. Ann Dermatol 201 1;23:239-241.

25 Marcoval J, Mana J, Moreno A, Gallego I, Fortuno Y, Peyri J: Foreign bodies in granulomatous cutaneous lesions of patients with sys temic sarcoidosis. Arch Dermatol 2001;137: 427-430.

26 Perez-Gala S, Delgado-Jimenez Y, Goiriz R, Fernandez-Herrera J, Fraga J, Garcia-Diez A: Cutaneous sarcoidosis limited to scars following pegylated interferon alfa and ribavirin therapy in a patient with chronic hepatitis $C$. Eur Acad Dermatol Venereol 2007;21:393 394.

27 Gregorio J, Meller S, Conrad C, Di Nardo A Homey B, Lauerma A, Arai N, Gallo RL, Digiovanni J, Gilliet M: Plasmacytoid dendritic cells sense skin injury and promote wound healing through type I interferons. J Exp Med 2010;207:2921-2930.

28 Gilliet M, Cao W, Liu YJ: Plasmacytoid dendritic cells: sensing nucleic acids in viral infection and autoimmune diseases. Nat Rev Immunol 2008;8:594-606.

29 Roche B, Samuel D: 'Transplantation: steroid use in HCV-infected liver transplant recipients. Nat Rev Gastroenterol Hepatol 2009;6: 198-200.

30 Oudghiri B, Benzagmout M, BoujrafS, Belahcen F, Ibrahimi A: Multisystem sarcoidosis in a patient on interferon-alpha therapy for chronic hepatitis C. J Glob Infect Dis 2012;4: 128-131.

31 Benzagmout M, Oudghiri B, Boujraf S, Chakour K, Chaoui Mel F: Fatal neurosarcoidosis induced by interferon alpha treatment in chronic hepatitis C. Neurol India 2010;58. 949-950.

32 Abdi EA, Nguyen GK, Ludwig RN, Dickout W): Pulmonary sarcoidosis following interferon therapy for advanced renal cell carcinoma. Cancer 1987;59:896-900.
33 Liozon E, Cransac M, Remenieras L, CartryThomas I, Vovor A, Bordessoule D: La sarcoïdose: une nouvelle complication de traitement par l'interféron alpha. Rev Med Interne 1993:14:1160.

34 Ohhata I, Ochi T, KurebayashiS, Masutani H, Kikui M: A case of subcutaneous sarcoid nodules induced by interferon-alpha (in Japanese). Nihon Kyobu Shikkan Gakkai Zasshi 1994;32:996-1000.

35 Bobbio-Pallavicini E, Valsecchi C, Tacconi F Moroni M, Porta C: Sarcoidosis following beta-interferon therapy for multiple myeloma. Sarcoidosis 1995;12:140-142.

36 Nakajima M, Kubota Y, Miyashita N, Niki Y Matsushima T, Manabe T: Recurrence of sarcoidosis following interferon alpha therapy for chronic hepatitis C. Intern Med 1996;35: 376-379.

37 Teragawa $H$, Hondo $T$, Takahashi $K$, Watanabe $\mathrm{H}$, Ohe $\mathrm{H}$, Hattori $\mathrm{N}$, Watanabe $\mathrm{Y}$, Amano $H$, Hino $F$, Ohbayashi $M$, Urushihara $T$, Yonehara S: Sarcoidosis after interferon therapy for chronic active hepatitis $C$. Intern Med 1996:35:19-23.

38 Otte HG, Hartig C, Stadler R: Sarcoidosis in interferon-alpha therapy (in German). Hautarzt 1997;48:482-487.

39 Hoffmann RM, Jung MC, Motz R, Gossl C, Emslander HP, Zachoval R, Pape GR: Sarcoidosis associated with interferon-alpha therapy for chronic hepatitis C. J Hepatol 1998;28:1058-1063.

40 Kikawada M, Ichinose Y, Kunisawa A, Yanagisawa N, Minemura K, Kasuga I, Yonemaru M, Kawanishi K, Takasaki M, Toyama K: Sarcoidosis induced by interferon therapy for chronic myelogenous leukaemia. Respirology 1998;3:41-44.

41 Mehta CL, Tyler RJ, Cripps DJ: Granulomatous dermatitis with focal sarcoidal features associated with recombinant interferon beta1b injections. J Am Acad Dermatol 1998;39: 1024-1028.

42 Yavorkovsky LL, Carrum G, Bruce S, McCarthy PL Jr: Cutaneous sarcoidosis in a patient with Philadelphia-positive chronic myelogenous leukemia treated with interferon-alpha. Am J Hematol 1998;58:80-81.

43 Eberlein-Konig B, Hein R, Abeck D, Engst R, Ring J: Cutaneous sarcoid foreign body granulomas developing in sites of previous skin injury after systemic interferon-alpha treatment for chronic hepatitis $\mathrm{C}$. $\mathrm{Br}$ ) Dermatol 1999;140:370-372.

44 Pietropaoli A, Modrak J, Utell M: Interferonalpha therapy associated with the development of sarcoidosis. Chest 1999;116:569-572.

45 Cacoub P, Sbai A, Frances C, Genesti C, Hausfater P, Piette JC: Systemic sarcoidosis during interferon-alpha therapy for chronic hepatitis $C$ virus infection (in French). Gastroenterol Clin Biol 2000;24:364-366. 
46 Savoye G, Goria O, Herve S, Riachi G, Noblesse I, Bastien L, Courville P, Lerebours E. Probable cutaneous sarcoidosis associated with combined ribavirin and interferon-alpha therapy for chronic hepatitis C (in French). Gastroenterol Clin Biol 2000;24:679.

47 Vander Els NI, Gerdes H: Sarcoidosis and IFN-alpha treatment. Chest 2000;117:294.

48 Pohl J, Stremmel W, Kallinowski B: Pulmonal sarcoidosis: a rare side effect of interferon-alpha treatment for chronic hepatitis $C$ infection (in German). Z Gastroenterol 2000;38: 951-955.

49 Frankova H, Gaja A, Hejlova N: Pulmonary sarcoidosis in a patient with essential thrombocythemia treated with interferon alpha: a short case report. Med Sci Monit 2000;6:380 382.

50 Fiorani C, Sacchi S, Bonacorsi G, Cosenza M: Systemic sarcoidosis associated with interferon-alpha treatment for chronic myelogenous leukemia. Haematologica 2000;85 1006-1007.

51 Vallina E, Garcia Diez A, Gallego $M$, Villaverde P, Rippe ML, Arribas JM: A case of pulmonary sarcoidosis induced by interferon alfa treatment in a female patient with hepatitis C (in Spanish). An Med Interna 2000;17: 538-539.

52 Neglia V, Sookoian S, Herrera $M$, Abeldano A, Kien MC, Chouela E, Frider B: Development of cutaneous sarcoidosis in a patient with chronic hepatitis $C$ treated with interfer on alpha 2b. I Cutan Med Surg 2001;5:406408

53 Miwa H, Furuya T, Tanaka S, Mizuno Y: Neurosarcoidosis associated with interferon therapy. Eur Neurol 2001;45:288-289.

54 Krehmeier H, Egberts EH: Sarcoidosis after interferon-alpha treatment in hepatitis C (in German). Dtsch Med Wochenschr 2001;126 460.

55 Ravenel JG, McAdams HP, Plankeel JF, Butnor KJ, Sporn TA: Sarcoidosis induced by interferon therapy. AJR Am J Roentgenol 2001; 177:199-201.

56 Husa P, Klusakova I, Jancikova I, Husova L, Horalek F: Sarcoidosis associated with interferon-alpha therapy for chronic hepatitis B. Eur J Intern Med 2002;13:129-131.

57 Wendling J, Descamps V, Grossin M, Marcellin P, Le Bozec P, Belaich S, Crickx B: Sarcoidosis during combined interferon alfa and ribavirin therapy in 2 patients with chronic hepatitis C. Arch Dermatol 2002;138:546547

58 Perez-Alvarez R, Perez-Lopez R, Lombrana JL, Rodriguez M, Rodrigo L: Sarcoidosis in two patients with chronic hepatitis $C$ treated with interferon, ribavirin and amantadine. J Viral Hepat 2002;9:75-79.

59 Nawras A, Alsolaiman MM, Mehboob S, Bartholomew C, Maliakkal B: Systemic sarcoidosis presenting as a granulomatous tattoo reaction secondary to interferon-alpha treatment for chronic hepatitis $C$ and review of the literature. Dig Dis Sci 2002;47:1627-1631.
60 Noguchi K, Enjoji M, Nakamuta M, Sugimoto R, Kotoh K, Nawata H: Various sarcoid le sions in a patient induced by interferon therapy for chronic hepatitis C. I Clin Gastroenterol 2002;35:282-284.

61 Gitlin N: Manifestation of sarcoidosis during interferon and ribavirin therapy for chronic hepatitis C: a report of two cases. Eur J Gastroenterol Hepatol 2002;14:883-885.

62 Yamada S, Mine S, Fujisaki T, Ohnari N, Eto S, Tanaka Y: Hepatic sarcoidosis associated with chronic hepatitis C. J Gastroentero 2002;37:564-570.

63 Li SD, Yong S, Srinivas D, Van Thiel DH: Reactivation of sarcoidosis during interferon therapy. J Gastroenterol 2002;37:50-54.

64 Leveque L, de Boulard A, Bielefeld P, Sgro C Hillon $P$, Gabreau T, Champigneulle B, Mak ki $\mathrm{H}$, Besancenot JF: Sarcoidosis during the treatment of hepatitis $C$ by interferon-alpha and ribavirin: 2 cases (in French). Rev Med Interne $2001 ; 22: 1248-1252$

65 Luchi S, Scasso A: Sarcoidosis, chronic hepatitis $C$ and interferon-alpha: two cases. Scand I Infect Dis 2003;35:775-776.

66 Tahan V, Ozseker F, Guneylioglu D, Baran A Ozaras R, Mert A, Ucisik AC, Cagatay T, Yilmazbayhan D, Senturk H: Sarcoidosis after use of interferon for chronic hepatitis $\mathrm{C}$ : report of a case and review of the literature. Dig Dis Sci 2003;48:169-173.

67 Rubinowitz AN, Naidich DP, Alinsonorin C Interferon-induced sarcoidosis. I Comput Assist 'Tomogr 2003;27:279-283.

68 Leclerc S, Myers RP, Moussalli J, Herson S, Poynard T, Benveniste O: Sarcoidosis and interferon therapy: report of five cases and review of the literature. Eur J Intern Med 2003; 14:237-243.

69 Tortorella C, Napoli N, Panella E, Antonaci A, Gentile A, Antonaci S: Asymptomatic systemic sarcoidosis arising 5 years after IFN-alpha treatment for chronic hepatitis $\mathrm{C}$ : a new challenge for clinicians. J Interferon Cytokine Res 2004;24:655-658.

70 Salvio A, Mormile M, Giannattasio F, Varriale M, d'Errico T, Balzano B, Carratu P, Tufano G, Visconti M: Pulmonary sarcoidosis during interferon therapy: a rare or underestimated event? Ann Ital Med Int 2004;19:58-62.

71 Menon Y, Cucurull E, Reisin E, Espinoza LR Interferon-alpha-associated sarcoidosis responsive to infliximab therapy. Am J Med Sc 2004;328:173-175.

72 Werchniak AE, Cheng SX, Dhar AD, Klaus SN: Sarcoidosis presenting as tattoo changes in a patient undergoing treatment with interferon-alpha and ribavirin. Clin Exp Dermatol 2004;29:547-548

73 Marzouk K, Saleh S, Kannass M, Sharma OP Interferon-induced granulomatous lung disease. Curr Opin Pulm Med 2004;10:435-440.

74 Rogers CJ, Romagosa R, Vincek V: Cutaneous sarcoidosis associated with pegylated interferon alfa and ribavirin therapy in a patien with chronic hepatitis C.J Am Acad Dermatol 2004;50:649-650.
75 Massaguer S, Sanchez M, Castel T: Mediastinal sarcoidosis induced by high-dose alpha-2-interferon therapy in a patient with malignant melanoma. Eur Radiol 2004;14: 1716-1717.

76 Alfageme Michavila I, Merino Sanchez $M$, Perez Ronchel J, Lara Lara I, Suarez Garcia E, Lopez Garrido I: Sarcoidosis following combined ribavirin and interferon therapy: a case report and review of the literature (in Spanish). Arch Bronconeumol 2004;40:45-49.

77 Papaioannides D, Fotinou M, Korantzopoulos P, Latsi P, Sinapidis D, Akritidis N, Orphanidou D: Sarcoidosis associated with interferon-alpha therapy for chronic hepatitis C. Med Sci Monit 2004;10:CS5-CS7.

78 Guilabert A, Bosch X, Julia M, Iranzo P, Mascaro JM Jr: Pegylated interferon alfa-induced sarcoidosis: two sides of the same coin. $\mathrm{Br} \mathrm{J}$ Dermatol 2005;152:377-379.

79 Tsiodras S, Drimala P, Stavreas N, Dachlythras M, Alamani AM, Naoim G: Uterine sarcoidosis potentially associated with interferon-alpha use for chronic hepatitis $\mathrm{C}$ infection. Obstet Gynecol 2005;105:1198-1201.

80 Butnor KJ: Pulmonary sarcoidosis induced by interferon-alpha therapy. Am J Surg Pathol 2005;29:976-979.

81 Celik G, Sen E, Ulger AF, Kumbasar OO, Bozkaya H, Alper D, Karayalcin S: Sarcoidosis caused by interferon therapy. Respirology 2005; 10:535-540.

82 Hirano A, Kataoka M, Nakata Y, Takeda K, Kamao T, Hiramatsu J, Kimura G, Tanimoto Y, Kanehiro A, Tanimoto M: Sarcoidosis occurring after interferon-alpha therapy for chronic hepatitis C: report of two cases. Respirology 2005;10:529-534.

83 Weyer P, Cummings OW, Knox KS: A 49-year-old woman with hepatitis, confusion, and abnormal chest radiograph findings. Chest 2005;128:3076-3079.

84 Akay BN, Ekmekci P, Sanli H, Celik G, Bozdayi $M$ : Cutaneous, pulmonary and hepatic sarcoidosis associated with autoimmune complications during interferon-alpha treatment for hepatitis $C$ virus infection. I Eur Acad Dermatol Venereol 2006;20:442-445.

85 Goldberg HJ, Fiedler D, Webb A, Jagirdar J, Hoyumpa AM, Peters J: Sarcoidosis after treatment with interferon-alpha: a case series and review of the literature. Respir Med 2006; 100:2063-2068.

86 Benali $S$, Boustière C, Castellani P, Cesari C, Jullien $M$, Lecomte $L$, Lebars $O$, Lambot $G$, Loyer R, Masseboeuf A, Perrier H, Oules V, Bourlière M: Sarcoidosis following pegylated interferon therapy: two cases (in French). Gastroenterol Clin Biol 2006;30:615-619.

87 Bolukbas C, Bolukbas FF, Kebdir T, Canayaz L, Dalay AR, Kilic G, Ovunc O: Development of sarcoidosis during interferon alpha $2 b$ and ribavirin combination therapy for chronic hepatitis $C-$ a case report and review of the literature. Acta Gastroenterol Belg 2005;68: $432-434$. 
88 Doyle MK, Berggren R, Magnus JH: Interferon-induced sarcoidosis. J Clin Rheumatol 2006;12:241-248.

89 Bonnet F, Morlat P, Dubuc J, De Witte S, Bonarek M, Bernard N, Lacoste D, Beylot J: Sarcoidosis-associated hepatitis $\mathrm{C}$ virus infection. Dig Dis Sci 2002;47:794-796.

90 Godoy E, Arora K, Atten MI, Youg S, Attar BM: Sarcoidosis induced by interferon-alpha therapy for chronic hepatitis C. Am J Gastroenterol 2001;96:S202.

91 Alazemi S, Campos MA: Interferon-induced sarcoidosis. Int I Clin Pract 2006;60:201-211.

92 Iwashita $M$, Maeda T, Tagami A, Watanabe N, Takada J, Asano T, Hatakeyama H, Hayashi T, Nishiwaki S, Saito K: A case of cardiac sarcoidosis occurring during combination therapy by IFN alpha and ribavirin for chronic hepatitis C (in Japanese). Nihon Shokakibyo Gakkai Zasshi 2010;107:1319-1327.

93 Bitetto D, Fumolo E, Fabris C, Toniutto P: Sarcoidosis or foreign-body granulomatous reaction during interferon treatment? Liver Int 2010;30:1083-1084.

94 Meller S, Reifenberger J, Ruzicka T, Homey B: Induktion einer Sarkoidose unter Therapie mit pegyliertem Interferon-alpha2a. Hautarzt 2006;57:317-318.

95 Honsová E, Sticová E, Sperl J: Cutaneous sarcoidosis during pegylated interferon alpha and ribavirin treatment of chronic hepatitis C - a case report (in Czech). Cesk Patol 2007;43:27-30.

96 Sanchez-Ruano J), Artaza-Varasa T, GomezRodriguez R, Gonzalez de Frutos C, RepisoOrtega A, Alcantara-Torres M: Cutaneopulmonary sarcoidosis during pegylated interferon plus ribavirin therapy (in Spanish). Gastroenterol Hepatol 2006;29:150.

97 Song $K$, Das $K$ : Cutaneous sarcoidosis in a woman with chronic hepatitis $C$ and interferon therapy: a case report. Acta Cytol 2010; 54:863-866.
98 Adla M, Downey KK, Ahmad J: Hepatic sarcoidosis associated with pegylated interferon alfa therapy for chronic hepatitis $\mathrm{C}$ : case report and review of literature. Dig Dis Sci 2008;53:2810-2812.

99 Doycheva D, Deuter C, Stuebiger N, Zierhut $\mathrm{M}$ : Interferon-alpha-associated presumed ocular sarcoidosis. Graefes Arch Clin Exp Ophthalmol 2009;247:675-680.

100 Yan KK, Dinihan I, Freiman I, Zekry A: Sarcoidosis presenting with granulomatous uveitis induced by pegylated interferon and ribavirin therapy for hepatitis C. Intern Med J 2008;38:207-210.

101 Lopez V, Molina I, Monteagudo C, Jorda E: Cutaneous sarcoidosis developing after treatment with pegylated interferon and ribavirin: a new case and review of the literature. Int J Dermatol 2011;50:287-291.

102 Petousi N, Thomas EC: Interferon-beta-in duced pulmonary sarcoidosis in a 30 -yearold woman treated for multiple sclerosis: a case report. J Med Case Rep 2012;6:344.

103 Chakravarty SD, Harris ME, Schreiner AM, Crow MK: Sarcoidosis triggered by interferon-beta treatment of multiple sclerosis: a case report and focused literature review. Semin Arthritis Rheum 2012;42:206-212.

104 Carbonelli C, Montepietra S, Caruso A Cavazza A, Feo C, Menzella F, Motti L, Zucchi L: Sarcoidosis and multiple sclerosis: systemic toxicity associated with the use of interferon-beta therapy. Monaldi Arch Chest Dis 2012;77:29-31.

105 O'Reilly S, White A, Florian A: Mediastinal sarcoidosis in a patient receiving interferonbeta for multiple sclerosis. Chest 2005;128: $450 \mathrm{~S}$.

106 Novoa R, Barnadas MA, Torras X, Curell R Alomar A: Foreign body granulomatous reaction to silica, silicone, and hyaluronic acid in a patient with interferon-induced sarcoidosis (in Spanish). Actas Dermosifiliogr 2012, E-pub ahead of print.

107 Albaker WI: Hypercalcemia induced by interferon therapy in chronic hepatitis C. I Family Community Med 2012;19:141144.
108 North J, Mully T: Alpha-interferon induced sarcoidosis mimicking metastatic melanoma. J Cutan Pathol 2011;38:585-589.

109 Morley AM, O’Sullivan E, Thaung C, Malhotra R: Sarcoid-related dacryoadenitis following treatment with interferon alpha and ribavarin for hepatitis C. Orbit 2011;30:2729.

110 Sionidou M, Spyratos D, Chloros D, Sichletidis L: Interferon a-induced sarcoidosis to a patient with polycythemia vera. BMJ Case Rep DOI: $10.1136 / \mathrm{bcr} .01 .2011 .3740$.

111 Cardoso C, Freire R, Alves A, Oliveira A: Interferon-induced sarcoidosis. BMJ Case Rep DOI: 10.1136/bcr.03.2011.3929.

112 Gayet AR, Plaisance P, Bergmann JF, Mouly $S$ : Development of sarcoidosis following completion of treatment for hepatitis $C$ with pegylated interferon-\{alpha\} $2 a$ and ribavirin: a case report and literature review. Clin Med Res 2010;8:163-167.

113 Heinzerling LM, Anliker MD, Muller J, Schlaeppi M, von Moos R: Sarcoidosis induced by interferon-alpha in melanoma patients: incidence, clinical manifestations, and management strategies. J Immunother 2010;33:834-839.

114 Rodriguez-Lojo R, Almagro M, Barja JM, Pineyro F, Perez-Varela L, Del Pozo I, Yebra-Pimentel MT, Fonseca E: Subcutaneous sarcoidosis during pegylated interferon alfa and ribavirin treatment for chronic hepatitis C. Dermatol Res Pract 2010;2010:230417.

115 Faurie P, Broussolle C, Zoulim F, Trepo C, Seve P: Sarcoidosis and hepatitis C: clinical description of 11 cases. Eur J Gastroenterol Hepatol 2010;22:967-972.

116 Le Bras M, Hervier B, Wastiaux H, Masseau A, Durant C, Jossic F, Hamidou M: Systemic sarcoidosis after treatment with pegylated interferon-alpha (in French). Rev Med Interne 2010;31:e11-e13.

117 Zampino MR, Corazza M, Borghi A, Marzola A, Virgili A: Hla typing in an IFNalpha-induced scar sarcoidosis: possible pathogenetic and clinical implications. Acta Derm Venereol 2009;89:661-662. 